\title{
Characterization and differentiation of body fluids, putrefaction fluid, and blood using Hounsfield unit in postmortem CT
}

\author{
Wolf-Dieter Zech • Christian Jackowski • \\ Yanik Buetikofer • Levent Kara
}

Received: 14 February 2014 / Accepted: 27 May 2014 / Published online: 6 June 2014

(C) Springer-Verlag Berlin Heidelberg 2014

\begin{abstract}
The purpose of the present study was to evaluate the ranges of Hounsfield unit (HU) found in body fluids, putrefaction fluids, and blood on postmortem CT and how these ranges are affected by postmortem interval, temperatures, and $\mathrm{CT}$ beam energy. Body fluids, putrefaction fluids, and blood from a total of 53 corpses were analyzed to determine the ranges of $\mathrm{HU}$ values from postmortem $\mathrm{CT}$ images that were taken prior to autopsy. The fluids measured in CT images were obtained at autopsy and examined in terms of macroscopic and microscopic appearances. Body fluids and blood were also collected in plastic bottles, which were subjected to $\mathrm{CT}$ scans at different beam energies $(80-130 \mathrm{kV})$ and at various fluid temperatures ( 4 to $40{ }^{\circ} \mathrm{C}$ ). At a postmortem interval of 1 to 4 days, the ranges of HU values of the serous fluids (13-38 HU) and the nonsedimented blood (40-88 HU) did not overlap. In the sedimented blood, the upper serum layer exhibited HU value ranges that overlapped with those of the serous fluids. The putrefaction fluids exhibited a range of $\mathrm{HU}$ values between 80 and $-130 \mathrm{HU}$. Elevated HU values were observed in fluids with accretive cell impurities. HU values decreased slightly with increasing temperature and CT beam energy. We concluded that serous fluids and blood in fresh corpses can be characterized and differentiated from each other based on $\mathrm{HU}$ value ranges. In contrast, body fluids in decomposed corpses cannot be differentiated by their HU value ranges. Different beam energies and corpse temperatures had only minor influences on $\mathrm{HU}$ value ranges and therefore
\end{abstract}

W.-D. Zech $(\bowtie) \cdot$ C. Jackowski $\cdot$ Y. Buetikofer $\cdot$ L. Kara Institute of Forensic Medicine, University of Bern, Buehlstrasse 20, 3012 Bern, Switzerland

e-mail: Wolf-Dieter.Zech@irm.unibe.ch should not be obstacles to the differentiation and characterization of body fluids and blood.

Keywords Computed tomographie · Post mortem imaging · HU measurements $\cdot$ Blood $\cdot$ Body fluids

\section{Introduction}

Postmortem computed tomography (PMCT) has become a useful tool as adjunct to forensic autopsy. Among other techniques, the use of cross-sectional PMCT images is feasible for the detection of blood and fluid collections [1-5]. The characterization and differentiation of body fluids and blood within a corpse can be of crucial importance for determining the cause of death or clarifying other relevant forensic issues [6-12]. In clinical CT images, body fluids are characterized and differentiated based on Hounsfield unit (HU) magnitudes and ranges [13-15]. In the Hounsfield scale, the radiodensity of distilled water at standard pressure and temperature is defined as zero $\mathrm{HU}$, and the radiodensity of air is defined as $-1,000 \mathrm{HU}$. In vivo, different tissues and fluids possess characteristic HU values that are determined by their radiodensities. The radiodensity of blood is known to range between 40 and $60 \mathrm{HU}$, and the exact $\mathrm{HU}$ value depends mainly on the cellular content. The radiodensities of serous fluids range between 15 and $30 \mathrm{HU}$, and the exact $\mathrm{HU}$ value depends on the protein content [16]. However, PMCT is vastly different from clinical CT due to various postmortem phenomena, such as putrefaction, biochemical degradation, and diffusion processes $[1-4,17]$. The radiodensities of fluids are known to depend on temperature and beam energy [18, 19]. PMCT scans are conducted at various body temperatures and with different CT beam energies. Currently, it is unknown how postmortem degradation processes, putrefaction and variations in body temperatures, and CT beam energies influence 
the radiodensities and HU values of body fluids and blood. It is possible that the known HU values for the fluids and blood of the living are not applicable to fluid media in corpses. Therefore, the goal of the present study was to evaluate Hounsfield unit ranges of body fluids, putrefaction fluids, and blood in postmortem CT in terms of cadaver age, temperature, and beam energy.

\section{Materials and methods}

\section{Study subjects}

Body fluids (i.e., cerebrospinal fluid (CSF), serous fluids, urine, bile, and putrefaction fluid) and blood of a total of 53 corpses were analyzed in a prospective study. Inclusion criteria for corpses were the existence of fluid collections that were detectable in CT images and that were large enough to place five independent regions of interest (ROIs) for $\mathrm{HU}$ value measurements. Corpses with no detectable fluid collections in CT images were excluded. The postmortem intervals (PMIs) of corpses ranged from 1 day to several months. Table 1 gives information on all cases regarding the circumstances of death, state of decomposition, postmortem intervals, and the presence of body fluids. The state of decomposition was determined using the radiological alteration index (RAI) from Egger et al. [20]. For reasons of tissue preservation, all corpses were stored for several hours in cooling chambers before the CT scans. The body temperatures of the corpses were not assessed during the CT scans due to the expected limited temperature ranges after storage in the cooling chambers.

\section{CT imaging and image analysis}

All corpses underwent CT scans (Somatom Emotion 6, Siemens Medical Solution, Forchheim, Germany) prior to autopsy. The CT scan parameters were as follows: beam energy, 130 kilovolts $(\mathrm{kV})$; rotation time, 1,500 ms; kernel, B30; slice thickness, $1.25 \mathrm{~mm}$; and increment, $0.7 \mathrm{~mm}$ and the field of view was adapted to the size of the object. The analyses of and $\mathrm{HU}$ value measurements from the $\mathrm{CT}$ images were performed by one observer on a Leonardo Workstation (Siemens, Forchheim, Germany). To obtain HU values for fluids and blood from CT images, a total of five independent and nonoverlapping ROIs were created in three different areas in which fluids and blood were detected in axial CT images. The ROIs were placed over the entire ventro-dorsal expansion of the fluid collections. The sizes of the ROIs depended on the extensions of the fluid collections (Fig. 1a, b). In cases of visible segmentations of putrefaction fluids $(n=7)$ and blood $(n=21)$, the upper and lower layers were measured separately (Fig. 1c).
Autopsy and sample collection

Autopsies were authorized by the local authorities and performed by forensic pathologists immediately following CT scanning or on the following day. At autopsy, fluids were obtained from the following body cavities: CSF from the lateral brain ventricles $(n=37)$, serous fluids from the thoracic $(n=31)$ and abdominal cavities $(n=19)$, blood from the right heart ventricle $(n=36)$ and when present from the thoracic $(n=13)$ and abdominal cavities $(n=9)$, urine from the urinal bladder $(n=28)$, and bile from the gall bladder $(n=23)$. A total of 14 of the 53 corpses were highly putrefied. From these corpses, putrefaction fluids were obtained from thoracic $(n=12)$ and abdominal cavities $(n=9)$.

Macroscopic and microscopic analysis of sampled fluids

The fluids that were measured in the $\mathrm{CT}$ images were obtained at autopsy and examined immediately afterwards in terms of macroscopic (color and clarity) and microscopic (cell count) appearances. The fluids in the thoracic and abdominal cavities that were light yellow to light red in color and clear or slightly unclear in appearance and had low cell counts were characterized as serous fluid or as CSF when these fluids were found in the lateral brain ventricles. Blood was characterized by its typical red appearance and the presence of countless erythrocytes. Fluids in the putrefied corpses that were dark red to black in color, unclear in appearance and contained microscopic debris were characterized as putrefaction fluids. For microscopic examination, the specimens of the obtained body fluids were pipetted onto an object plate, and five different visual fields were viewed at $\times 20$ magnification. Cell counts were categorized as follows: no cells per visual field; fewer than 10 cells per visual field, between 10 and 20 cells per visual field; and more than 20 cells per visual field.

CT imaging of fluids in bottles at different temperatures and beam energies

CSF, serous fluids, and blood were collected in plastic bottles and underwent CT scans after autopsy. To determine the radiodensity of distilled water according to the CT scanner that was used, plastic bottles were also filled with distilled water and underwent CT scans. The CT scans in bottles were performed with different beam energies $(80,110$, and $130 \mathrm{kV})$ and at different fluid temperatures (ranging from 4 to $40{ }^{\circ} \mathrm{C}$ ). For the CT scans at different temperatures, the fluids, blood, and distilled water in the plastic bottles were cooled in a refrigerator and heated in a warming bath in $5{ }^{\circ} \mathrm{C}$ steps. The measurements of the $\mathrm{HU}$ values from the $\mathrm{CT}$ 
Table 1 Circumstances of death, state of decomposition according to the RA index (RAI) from Egger et al [20], postmortem intervals, and presence of body fluids in 53 investigated forensic cases

\begin{tabular}{|c|c|c|c|c|c|c|c|c|c|}
\hline Case no. & Manner and cause of death & PM delay (days) & RAI & Blood & $\mathrm{CSF}$ & Serous fluids & bile & Urine & Putrefaction fluid \\
\hline 1 & Natural-undetermined & $>20$ & 86 & - & - & - & - & - & + \\
\hline 2 & Natural-cardiac & $<1$ & 2 & + & + & + & - & + & - \\
\hline 3 & Natural-intracranial Hemorrhage & 3 & 18 & + & + & + & + & + & - \\
\hline 4 & Natural-pneumonia & 1 & 7 & + & + & + & - & - & - \\
\hline 5 & Suicide-hanging & 2 & 26 & + & + & - & + & + & - \\
\hline 6 & Natural-cardiac & $<1$ & 0 & + & + & + & + & + & - \\
\hline 7 & Suicide-hanging & $>30$ & 85 & - & - & - & - & - & + \\
\hline 8 & Natural-septic shock & 4 & 39 & + & + & + & + & + & - \\
\hline 9 & Homicide-gunshot & 2 & 36 & + & + & + & - & - & - \\
\hline 10 & Natural-aortic dissection & $<1$ & 6 & + & + & + & + & + & - \\
\hline 11 & Natural-cardiac & $>30$ & 100 & - & - & - & - & - & + \\
\hline 12 & Accident-exsanguination & 2 & 46 & - & + & - & - & + & - \\
\hline 13 & Natural-cardiac & 4 & 49 & + & + & + & + & - & - \\
\hline 14 & Accident-head trauma & $<1$ & 10 & + & + & + & + & + & - \\
\hline 15 & Natural-undetermined & $>40$ & 71 & - & - & - & - & - & + \\
\hline 16 & Accident-intoxication & 1 & 18 & + & + & + & + & - & - \\
\hline 17 & Suicide-gunshot & 1 & 0 & + & + & + & + & + & - \\
\hline 18 & Natural-cardiac & 3 & 12 & + & + & - & - & - & - \\
\hline 19 & Homicide-stabbing & $<1$ & 7 & + & + & + & + & + & - \\
\hline 20 & Natural-undetermined & $>30$ & 85 & - & - & - & - & - & + \\
\hline 21 & Natural-cerebal ischemia & 4 & 22 & + & + & + & - & + & - \\
\hline 22 & Suicide-hanging & 2 & 7 & + & + & + & - & + & - \\
\hline 23 & Suicide-intoxication & $>60$ & 100 & - & - & - & - & - & + \\
\hline 24 & Homicide-gunshot & 2 & 39 & + & + & + & + & + & - \\
\hline 25 & Natural-cardiac & $<1$ & 0 & + & + & + & + & + & - \\
\hline 26 & Accident-drowning & 2 & 40 & - & + & + & + & - & - \\
\hline 27 & Natural-cardiac & 3 & 11 & + & + & + & - & + & - \\
\hline 28 & Natural-undetermined & $>20$ & 91 & - & - & - & - & - & + \\
\hline 29 & Accident-positional asphyxia & 1 & 2 & + & + & + & + & + & - \\
\hline 30 & Accident-head trauma & 2 & 14 & + & + & + & - & - & - \\
\hline 31 & Accident-intoxication & 2 & 5 & + & + & + & + & + & - \\
\hline 32 & Suicide-hanging & 3 & 21 & + & - & - & + & + & - \\
\hline 33 & Natural-undetermined & $>14$ & 66 & - & - & - & - & - & + \\
\hline 34 & Natural-malignant tumor & $<1$ & 0 & + & + & + & + & + & - \\
\hline 35 & Suicide-electrocution & 4 & 28 & + & + & + & + & + & - \\
\hline 36 & Natural-pneumonia & 4 & 22 & + & + & - & - & - & - \\
\hline 37 & Accident-polytrauma & 1 & 18 & + & + & + & + & + & - \\
\hline 38 & Natural-cardiac & 1 & 0 & + & + & - & - & - & - \\
\hline 39 & Undetermined/undetermined & $>20$ & 70 & - & - & - & - & - & + \\
\hline 40 & Natural-cardiac & $>40$ & 100 & - & - & - & - & - & + \\
\hline 41 & Suicide-intoxication & 1 & 7 & + & + & + & + & + & - \\
\hline 42 & Accident-falling & 2 & 10 & + & + & + & - & - & - \\
\hline 43 & Accident-polytrauma & 2 & 18 & + & + & + & + & + & - \\
\hline 44 & Natural-cardiac & $<1$ & 0 & + & + & + & - & + & - \\
\hline 45 & Natural-sudden infant death & $<1$ & 2 & + & + & - & - & - & - \\
\hline 46 & Natural-cardiac & $>20$ & 61 & - & - & - & - & - & + \\
\hline 47 & Accident-head trauma & 1 & 0 & + & + & + & + & + & - \\
\hline 48 & Natural-cardiac & $>40$ & 72 & - & - & - & - & - & + \\
\hline
\end{tabular}


Table 1 (continued)

\begin{tabular}{|c|c|c|c|c|c|c|c|c|c|}
\hline Case no. & Manner and cause of death & PM delay (days) & RAI & Blood & $\mathrm{CSF}$ & Serous fluids & bile & Urine & Putrefaction fluid \\
\hline 49 & Accident-intoxication & 2 & 7 & + & + & + & - & + & \\
\hline 50 & Natural-undetermined & $>20$ & 78 & - & - & - & - & - & + \\
\hline 51 & Accident-chest trauma & 3 & 15 & + & - & - & + & + & - \\
\hline 52 & Homicide-stabbing & $<1$ & 1 & - & + & + & - & + & - \\
\hline 53 & Suicide-gunshot & $>14$ & 61 & - & - & - & - & - & + \\
\hline
\end{tabular}

Note that all cases with detectable putrefaction fluids presented with a RAI over 60

images of the bottles were performed using a Leonardo workstation. For each bottle, six different ROI of the same size were created in each third of the bottle in the longitudinal plane (Fig. 1d).

\section{Results}

HU values of fluids and blood in corpses

Figure 2 shows the HU value ranges obtained for blood and the body fluids containing zero or very few cells from the fresh corpses and for the putrefaction fluids from the decomposed corpses. In the fresh corpses, the serous fluids and blood exhibited $\mathrm{HU}$ value ranges that did not overlap. In cases of sedimentation of the blood, the upper layers exhibited
HU values that overlapped with those of the serous fluids. In some cases, the putrefaction fluids exhibited negative $\mathrm{HU}$ values that were as low as $-70 \mathrm{HU}$. Sedimentation of the putrefaction fluid was observed in some corpses $(n=7)$ (Fig. 3). In these cases, the upper layer always exhibited negative $\mathrm{HU}$ values that ranged between -80 and $-130 \mathrm{HU}$, and the lower layer exhibited $\mathrm{HU}$ values that were similar to those of the blood from the fresh corpses.

The HU values of all serous fluids and CSF with accretive cell impurities were elevated (Fig. 4).

HU values of fluids and distilled water in bottles at different temperatures and beam energies

Figure 5 illustrates the temperature dependence of the $\mathrm{HU}$ values for the fluids and blood. The HU values of CSF, serous fluids, and blood decreased with increasing temperature.
Fig. 1 HU measurements of the fluid media in the CT images of corpses and plastic bottles. a Measurements of the HU values of fluid collections in thoracic cavity on axial CT images. Five ROIs were created in the axial images over the entire ventrodorsal extent of each fluid collection. b Measurements were taken at three different positions of the fluid collection (green lines in the sagittal CT image). $\mathbf{c}$ In cases of visible sedimentation of fluid media, the upper and lower layers were measured separately. d Measurement of the HU values of the fluid media filled in plastic bottles was accomplished with six different ROIs of the same size that were placed in each third of the bottle (green lines) in the longitudinal plane
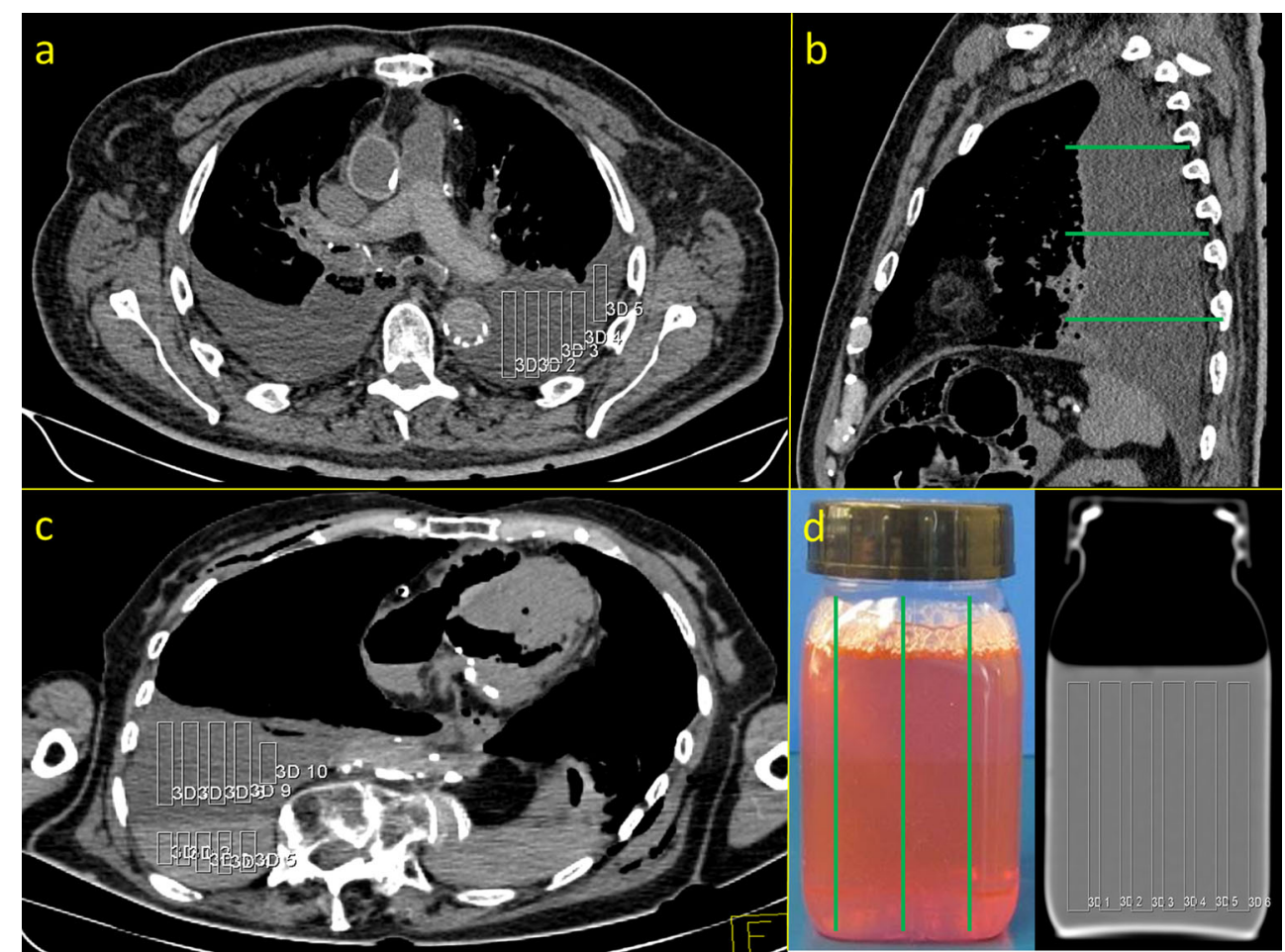


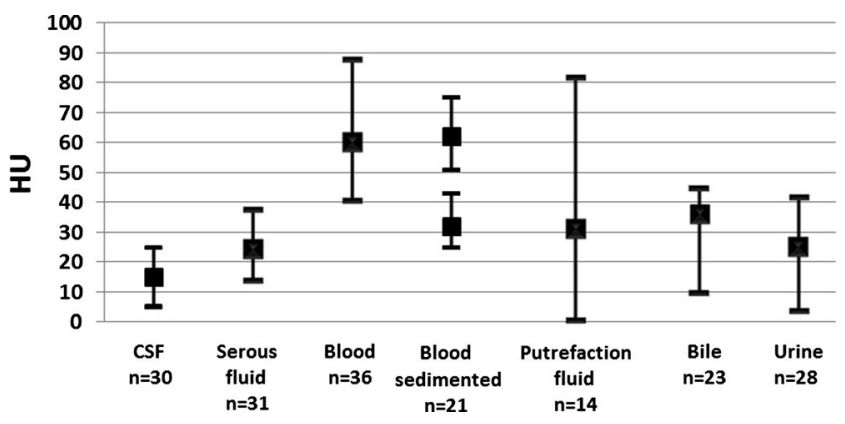

Fig. 2 Ranges (mean, minimum, and maximum, $130 \mathrm{kV}$ ) of the HU values found for blood, body fluids (with zero or very few cells), and putrefaction fluid of the corpses (negative HU values and sedimentation of the putrefaction fluids are not depicted). Postmortem intervals ranged from 1 to 4 days for the body fluids and blood to several weeks to several months for the putrefied corpses

Between 4 and $40{ }^{\circ} \mathrm{C}$, a maximum deviation of $10 \mathrm{HU}$ in the means of all of the investigated fluids and blood was observed (CSF: minimum of $15 \mathrm{HU}$, maximum of $25 \mathrm{HU}$; serous fluids: minimum of $20 \mathrm{HU}$, maximum of $30 \mathrm{HU}$; blood: minimum of $55 \mathrm{HU}$, maximum of $65 \mathrm{HU}$; distilled water: minimum of $-1 \mathrm{HU}$, maximum of $3 \mathrm{HU})$.

The HU values also depended on the beam energy of the CT scanner. Figure 6 shows that the mean HU values of the examined fluids and blood decreased at the higher beam energies of 110 and $130 \mathrm{kV}$. The maximum deviation of the means of $\mathrm{HU}$ values between 80 and $130 \mathrm{kV}$ was $10 \mathrm{HU}$ across all examined fluids and blood (CSF: minimum of $15 \mathrm{HU}$, maximum of $25 \mathrm{HU}$; serous fluids: minimum of $24 \mathrm{HU}$, maximum of $23 \mathrm{HU}$; blood: minimum of $60 \mathrm{HU}$, maximum of $70 \mathrm{HU}$; distilled water: minimum of $0 \mathrm{HU}$, maximum of $3 \mathrm{HU}$ ).

\section{Discussion}

The results of the present study indicate that at short postmortem intervals of a few days, the serous body fluids and blood

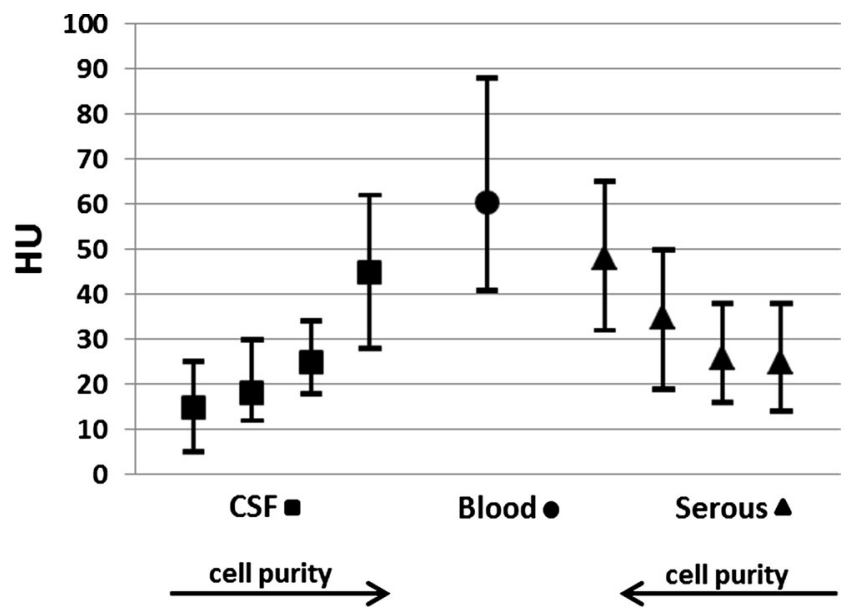

Fig. 4 Ranges of HU values (mean, minimum, and maximum, $130 \mathrm{kV}$ ) for CSF $(n=37)$ and serous fluids $(n=50)$ with increasing levels of cellular impurity (i.e., no cells, fewer than 10 cells, fewer than 20 cells, and more than 20 cells per visual field at $\times 20$ magnification) compared to that of nonsedimented blood $(n=36)$. Note that the HU values of the CSF and serous fluids increase with increasing cellular impurity

retain $\mathrm{HU}$ values that are similar to the known characteristic HU values of these fluids in living [16]. Therefore, serous fluids and blood can be differentiated based on HU values in fresh corpses. The differentiation of blood and serous fluids is highly relevant for forensic postmortem imaging. For example, evidence of large volumes of blood in the thoracic cavities or the abdominal cavity may be indicative of exsanguination or traumatic lesions to the relevant organs [6-12]. In such cases, the detection of blood collections may also be relevant for the decision to perform PMCT-angiography to detect the source of exsanguination [21, 22]. In contrast, equal volumes of serous fluid in the thoracic cavities or the abdominal cavity often suggest other causes of death because such fluid distributions are the consequence of chronic or malignant diseases [23-27].

In postmortem CT images, blood can be sedimented due to the cessation of blood circulation. In such cases, blood is easy for the image reader to detect because serous fluids do not
Fig. 3 Ranges of the HU values (mean, minimum, and maximum, $130 \mathrm{kV}$ ) of not sedimented putrefaction fluids and sedimented putrefaction fluids (a). Note that putrefaction fluids can exhibit negative HU values. In cases of sedimentation, the upper layers exhibited negative HU values (b, segmented putrefaction fluid in the right thoracic cavity with an upper hypodense fatty layer (solid arrow) and a lower hyperdense fluid layer (dashed arrow))

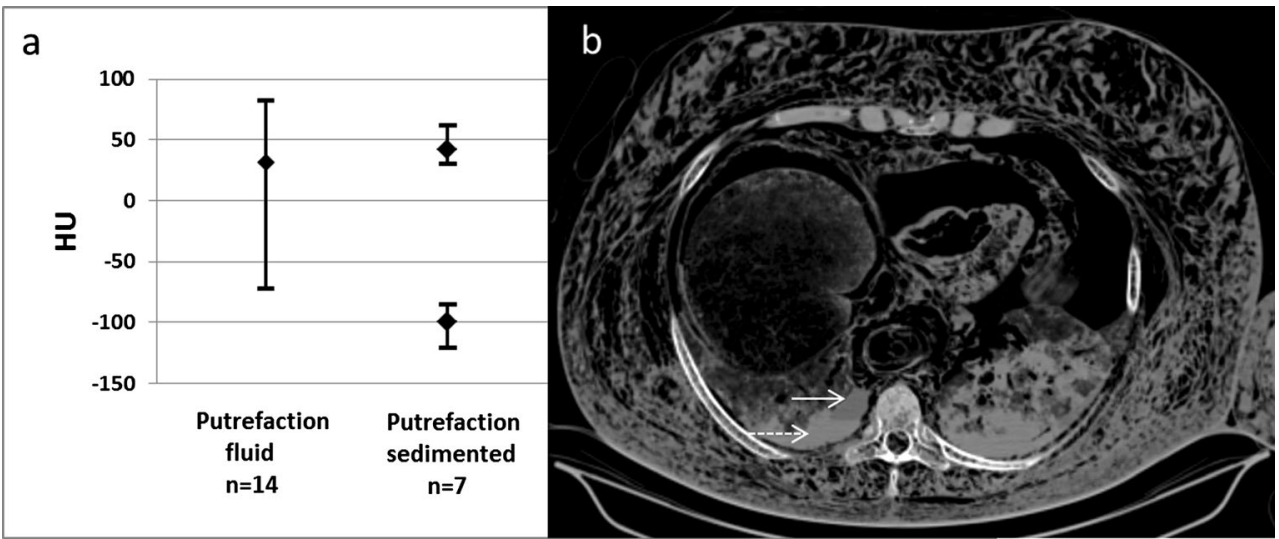




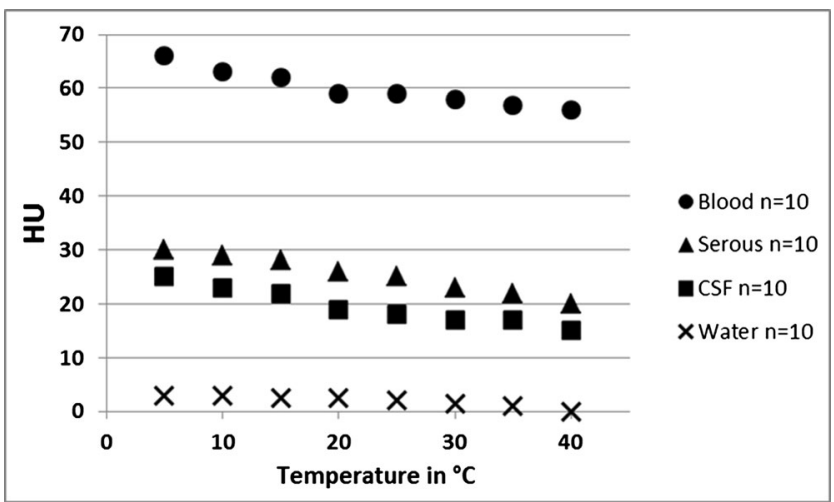

Fig. 5 Mean HU values for blood, CSF, and serous fluids that were filled in plastic bottles and scanned at temperatures that ranged from 4 to $40{ }^{\circ} \mathrm{C}$ (beam energy, $130 \mathrm{kV}$ ). Note that, in all media, the HU values decreased with increasing temperature

appear to be sedimented $[5,16]$. However, sedimentation of the blood is not always present in postmortem CT images. In such cases, blood and serous fluids can only be differentiated based on their HU value ranges. Furthermore, early in the process of sedimentation, a gray value contrast between the upper and lower layers of the sedimented blood may not yet be visible on the CT images. Therefore, we recommend that ROIs in the CT images be placed over the entire ventrodorsal expansions of fluid collections and not be limited to the upper or lower portions. Such ROI placement will prevent misinterpretation of the upper portions of blood collections as purely serous fluid collections. The present study verified that if body fluids of fresh corpses exhibit $\mathrm{HU}$ values below 40, the existence of blood can be excluded. Hence, fluids with HU values above 40 can be characterized as blood or fluids with relevant impurities due to blood cells. However, this rule is not applicable for cases in which an antemortem CT scan with an injection of contrast agent has been performed. The increases of the $\mathrm{HU}$ values of serous fluids that accompany increases in

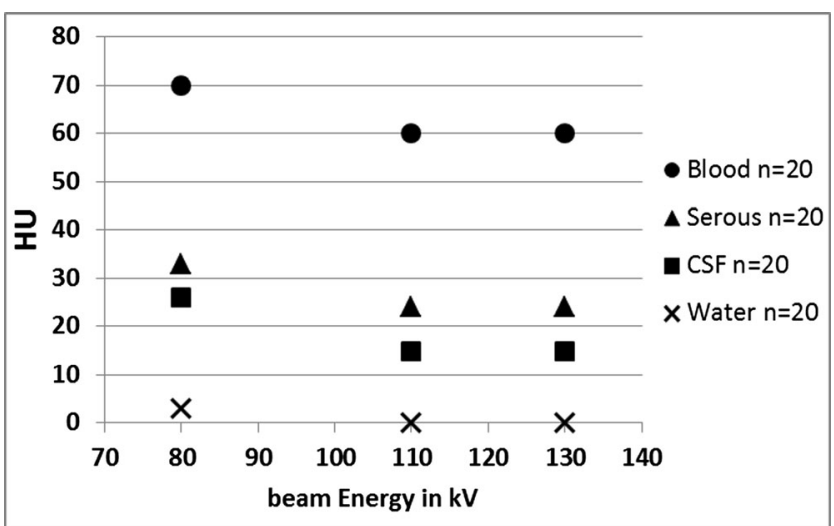

Fig. 6 Mean HU values for blood, CSF, and serous fluids that were filled in plastic bottles and scanned with different beam energies $(80,110$, and $130 \mathrm{kV}$ ). Note that the scans at 110 and $130 \mathrm{kV}$ resulted in slightly lower HU values cellular impurities are attributable to increased radiodensities due to the corpuscular cellular components $[5,16,21,22,28]$.

For the first time, the present study evaluated the HU values of putrefaction fluid. This study demonstrated that putrefaction fluid exhibits $\mathrm{HU}$ values that range from +80 to $-130 \mathrm{HU}$. The existence of negative HU values is attributable to the presence of the fatty contents that can be a component of putrefaction fluid in decomposed corpses [29]. The presence of fatty contents in putrefaction fluid may also explain the phenomenon of sedimentation because all observed sedimented putrefaction fluids exhibited negative HU values in their upper layers that were typical of fatty tissue in the living [30]. Since putrefaction fluid can mimic serous fluids as well as blood, we conclude that HU measurements in decomposed corpses are not useful for the differentiation of body fluids. Therefore, it is useful to determine the state of decomposition in PMCT before interpreting the HU values of fluids. With the RA index from Egger et al., the state of decomposition can be identified in PMCT. When RA indices indicate advanced decomposition, the image reader has to consider that the body fluids cannot be interpreted based on their HU values [20]. In fact, in the present study, all corpses with detectable putrefaction fluids presented with an RAI over 60. It can be concluded that fluid collections in corpses with a RAI over 60 can be identified as putrefaction fluid but cannot be further interpreted by their $\mathrm{HU}$ values.

Forensic PMCT scans are regularly being conducted across a range of corpse temperatures that typically vary between 4 and $40{ }^{\circ} \mathrm{C}$, depending on the PMI and the condition at death (1-3). Temperature is known to influence radiodensity and HU values because fluid densities decrease with increasing temperature $[1,19]$. In the present study, $\mathrm{HU}$ values were observed to decrease with increasing temperature. However, the maximum deviation of the mean $\mathrm{HU}$ values across a temperature range of 4 to $40{ }^{\circ} \mathrm{C}$ was only $10 \mathrm{HU}$. Thus, variations in corpse temperature should not be an obstacle to the identification of body fluids and blood based on $\mathrm{HU}$ values. A limitation of the present study was that the body temperatures of corpses were not assessed at the time of the CT scan. Thus, no data for influence of the temperature on $\mathrm{HU}$ values of fluids in corpses are available. However, the physical properties of the investigated fluids and fluid mediums in bottles and corpses are the same. Hence, information on the temperature dependence of $\mathrm{HU}$ values gained from the fluid CT scans in bottles should be conferrable to the fluids in corpses.

In forensic practice, corpses are $\mathrm{CT}$ scanned with different beam energies that range from 80 to $130 \mathrm{kV}$ [1-3]. Beam energy is defined by the X-ray tube voltage setting of the CT scanner and is measured in kilovolts $[31,32]$. In the present study, HU values were observed to decrease with increasing beam energy. The maximum deviation of the mean $\mathrm{HU}$ values of the fluids scanned across the beam energy range of 80 to 
$130 \mathrm{kV}$ was $10 \mathrm{HU}$. Thus, the effect of beam energy across the range of regularly used beam energies on $\mathrm{HU}$ values can be regarded as relatively small and should not complicate the characterization and identification of body fluids and blood.

A previous study revealed that the mean $\mathrm{HU}$ values of the same nonorganic objects varied significantly across different CT scanners [33]. This variation was probably due to differences in the mean energies of each of the CT scanner at the same $\mathrm{kV}$ setting. However, the investigated objects were highly absorbent materials with radiodensities that ranged from 1,000 to $30,000 \mathrm{HU}$. It is highly likely that media with lower attenuations, such as organic fluids, do not exhibit relevant scanner-dependent variations in HU values. Therefore, the ranges of $\mathrm{HU}$ values for blood and body fluids reported in the present study should also be valid for other CT scanners.

\section{Conclusions}

In corpses with a postmortem interval of 1 to 4 days, the HE values of serous fluids and blood are similar to the known $\mathrm{HU}$ values for these fluids in the living. Hence, in postmortem CT images of fresh corpses, serous fluids and blood can be characterized and differentiated based on the ranges of their $\mathrm{HU}$ values. The HU value ranges of putrefaction fluids overlap with those of serous fluids and blood in fresh corpses. In decomposed corpses with a radiological alteration index over 60 , the interpretation of body fluids based on the ranges of their $\mathrm{HU}$ values is not expedient because these fluids can be determined as putrefaction fluids. Different beam energies and corpse temperatures had only minor influences on the $\mathrm{HU}$ value ranges and therefore should not complicate the differentiation and characterization of body fluids and blood.

Acknowledgments The authors would like to thank the team of forensic pathologists and forensic autopsy technicians at the Institute of Forensic Medicine Bern for their support in handling the cases.

\section{References}

1. Thali MJ, Yen K, Schweitzer W, Vock P, Boesch C, Ozdoba C, Schroth G, Ith M, Sonnenschein M, Doernhoefer T, Scheurer E, Plattner T, Dirnhofer R (2003) Virtopsy, a new imaging horizon in forensic pathology: virtual autopsy by postmortem multislice computed tomography (MSCT) and magnetic resonance imaging (MRI) - a feasibility study. J Forensic Sci 48(2):386-403

2. Knight B (1996) Forensic pathology. Arnold, London

3. Brogdon BG (2010) Brogdon's Forensic Radiology, 2nd edn. CRC Press, Boca Raton

4. Dirnhofer R, Jackowski C, Vock P, Potter K, Thali MJ (2006) Virtopsy: minimally invasive, imaging-guided virtual autopsy. Radiographics 26(5):1305-1033
5. Jackowski C, Thali M, Aghayev E, Yen K, Sonnenschein M, Zwygart K, Dirnhofer R, Vock P (2006) Postmortem imaging of blood and its characteristics using MSCT and MRI. Int J Legal Med 120(4):233-40

6. Aghayev E, Christe A, Sonnenschein M, Yen K, Jackowski C, Thali MJ, Dirnhofer R, Vock P (2008) Postmortem imaging of blunt chest trauma using CT and MRI: comparison with autopsy. J Thorac Imaging 23(1):20-7

7. Bolliger SA, Thali MJ, Aghayev E, Jackowski C, Vock P, Dirnhofer R, Christe A (2007) Postmortem noninvasive virtual autopsy: extrapleural hemorrhage after blunt thoracic trauma. Am J Forensic Med Pathol 28(1):44-7

8. Aghayev E, Sonnenschein M, Jackowski C, Thali M, Buck U, Yen K, Bolliger S, Dirnhofer R, Vock P (2006) Postmortem radiology of fatal hemorrhage: measurements of cross-sectional areas of major blood vessels and volumes of aorta and spleen on MDCT and volumes of heart chambers on MRI. AJR 187:209-215

9. Burke M, Parsons S, Bassed R (2012) Management of medicolegal natural deaths from hemopericardium or hemothorax using postmortem CT scanning. Forensic Sci Med Pathol 8(4): 367-72

10. Makhlouf F, Scolan V, Ferretti G, Stahl C, Paysant F (2013) Gunshot fatalities: correlation between post-mortem multi-slice computed tomography and autopsy findings: a 30-months retrospective study. Leg Med (Tokyo) 15(3):145-8

11. Yamazaki K, Shiotani S, Ohashi N, Doi M, Kikuchi K, Nagata C, Honda K (2006) Comparison between computed tomography (CT) and autopsy findings in cases of abdominal injury and disease. Forensic Sci Int 162(1-3):163-6

12. Riezzo I, Di Battista B, De Salvia A, Cantatore S, Neri M, Pomara C, Turillazzi E, Fineschi V (2014) Delayed splenic rupture: dating the sub-capsular hemorrhage as a useful task to evaluate causal relationships with trauma. Forensic Sci Int 234:64-71

13. Allen BC, Barnhart H, Bashir M, Nieman C, Breault S, Jaffe TA (2012) Diagnostic accuracy of intra-abdominal fluid collection characterization in the era of multidetector computed tomography. Am Surg 78(2):185-9

14. Bydder GM, Kreel L (1980) Attenuation values of fluid collections within the abdomen. J Comput Assist Tomogr 4(2):145-50

15. Churchill RJ (1989) CT of intra-abdominal fluid collections. Radiol Clin North Am 27(4):653-66

16. Huda W, Slone R (2003) Review of Radiologic Physics, 3rd edn. Lippincott Williams \& Wilkins, Philadelphia

17. Christe A, Flach P, Ross S, Spendlove D, Bolliger S, Vock P, Thali MJ (2010) Clinical radiology and postmortem imaging (Virtopsy) are not the same: specific and unspecific postmortem signs. Leg Med (Tokyo) 12(5):215-22

18. Schmitt WG (1986) Energy dependence of Hounsfield numbers. Röfo 145(2):221-3

19. Bydder GM, Kreel L (1979) The temperature dependence of computed tomography attenuation values. J Comput Assist Tomogr 3(4): 506-10

20. Egger C, Vaucher P, Doenz F, Palmiere C, Mangin P, Grabherr S (2012) Development and validation of a postmortem radiological alteration index: the RA-Index. Int J Legal Med 126(4):559-66

21. Grabherr S, Grimm J, Dominguez A, Vanhaebost J, Mangin P (2014) Advances in post-mortem CT-angiography. Br J Radiol 87(1036): 20130488. doi:10.1259/bjr.20130488

22. Jackowski C, Persson A, Thali MJ (2008) Whole body postmortem angiography with a high viscosity contrast agent solution using poly ethylene glycol as contrast agent dissolver. J Forensic Sci 53(2):465-8

23. Montano-Loza AJ (2013) New concepts in liver cirrhosis: clinical significance of sarcopenia in cirrhotic patients. Minerva Gastroenterol Dietol 59(2):173-86 
24. Treglia G, Sadeghi R, Annunziata S, Lococo F, Cafarotti S, Bertagna F, Prior JO, Ceriani L, Giovanella L (2014) Diagnostic accuracy of (18)F-FDG-PET and PET/CT in the differential diagnosis between malignant and benign pleural lesions: a systematic review and metaanalysis. Acad Radiol 21(1):11-20

25. Solooki M, Miri M (2013) Approach to undiagnosed exudative pleural effusion: the diagnostic yield of blind pleural biopsy. Caspian J Intern Med 4(2):642-7

26. Huang XE, Wei GL, Huo JG, Wang XN, Lu YY, Wu XY, Liu J, Xiang J, Feng JF (2013) Intrapleural or intraperitoneal lobaplatin for treatment of patients with malignant pleural effusion or ascites. Asian Pac J Cancer Prev 14(4):2611-4

27. Adhikari P, Pathak UN, Uprety D, Sapkota S (2012) Profile of ascites patient admitted in Nepal Medical College Teaching Hospital. Nepal Med Coll J 14(2):111-3

28. Schleyer F (1958) Postmortem blood viscosity, blood cell volume, osmotic erythrocyte resistance and blood sedimentation in relation to cadaver age and cause of death. Virchows Arch 331(3):276-86

29. Brinkmann B, Madea B (2004) Handbuch gerichtliche Medizin, vol 1, 1st edn. Springer, Berlin

30. Makrogiannis S, Caturegli G, Davatzikos C, Ferrucci L (2013) Computer-aided assessment of regional abdominal fat with food residue removal in CT. Acad Radiol 20(11):1413-21

31. Weissleder R, Wittenberg J, Harisinghani MG, Chen JW (2011) Primer of diagnostic imaging, 5th edn. Mosby Elsevier, St. Louis

32. Strandberg S, Wretling ML, Wredmark T, Shalabi A (2010) Reliability of computed tomography measurements in assessment of thigh muscle cross-sectional area and attenuation. BMC Med Imaging 10-18. doi:10.1186/1471-2342-10-18

33. Ruder TD, Thali Y, Schindera ST, Dalla Torre SA, Zech WD, Thali MJ, Ross S, Hatch GM (2012) How reliable are Hounsfield-unit measurements in forensic radiology? Forensic Sci Int 220(1-3): $219-23$ 